\title{
Measurements and ERO simulations of carbon flows in the high-field side main SOL in AUG
}

\author{
T. Makkonen ${ }^{\mathrm{a}}$, M. Groth ${ }^{\mathrm{a}}$, M. I. Airila ${ }^{\mathrm{b}}$, R. Dux ${ }^{\mathrm{c}}$, A. Janzer ${ }^{\mathrm{c}}$, T. Kurki-Suonio ${ }^{\mathrm{a}}$, T. Lunt ${ }^{\mathrm{c}}$, \\ H.W. Mueller ${ }^{\mathrm{c}}$, T. Puetterich ${ }^{\mathrm{c}}$, E. Viezzer ${ }^{\mathrm{c}}$ and the ASDEX Upgrade team \\ ${ }^{a}$ Aalto University School of Science and Technology, Association Euratom-Tekes \\ ${ }^{b}$ VTT Technical Research Centre of Finland, Association Euratom-Tekes \\ ${ }^{c}$ Max-Planck-Institut fur Plasmaphysik, EURATOM Association
}

The plasma flow in the SOL is important for impurity migration. There are few measurements from the HFS main SOL. To study the flow in the HFS SOL of ASDEX Upgrade, methane was injected from a HFS valve. The ensuing carbon emission distribution was followed using a fast video camera and a spectrometer was used to determine the Doppler shift of the injected carbon. This was carried out in lower single null L-mode and H-mode for low and high density plasmas. To elucidate the coupling between the flow of the injected carbon and the actual deuterium flow, ERO simulations were utilized. A strong carbon and deuterium flow towards the lower inner divertor was observed for high density plasmas in the far SOL along with a shear in the flow profile.

\section{PACS: 52.25.Vy, 52.65.Cc, 52.70.Kz}

PSI-20 Keywords: ASDEX-Upgrade, Carbon-Impurities, Edge Plasma, Plasma flow, Spectroscopy Corresponding Author Address: Aalto University, P.O. Box 14100, FI-00076 AALTO, Finland Corresponding Author e-mail: toni.makkonen@aalto.fi

Presenting Author: Toni Makkonen

Presenting Author e-mail: toni.makkonen@aalto.fi 


\section{Introduction}

Both the poloidal and radial flow profiles in the scrape off layer (SOL) of tokamaks are important for impurity transport [1][2] and as a boundary condition for the core plasma. Impurity transport is related to material migration, tritium co-deposition, and core contamination - issues that are crucial for ITER and commercial fusion power. The radial profiles of the SOL flow has been measured in ASDEX Upgrade (AUG) close to the x-point for both divertor legs using a reciprocating Langmuir probe [3] and spectroscopy [4], and in the low field side (LFS) midplane using a Langmuir probe [5]. Also, charge exchange recombination spectroscopy (CXRS) core rotation measurements exists for both the HFS [6] and LFS [7]. The flow profile in the SOL is important for plasma-wallinteraction studies currently carried out in AUG [8][9][10], and we therefore investigated the radial flow profile in the HFS SOL - a new poloidal position in AUG.

The measurements were done by injecting methane and following the ensuing low charge state carbon emission with a fast video camera and a spectrometer. The experimental technique is inspired by C-Mod experiments [11][12], where the deuterium flow in the HFS SOL has also been measured using a Langmuir probe [13]. The reason for measuring injected carbon with the spectrometer is that standard CXRS methods rely on fully stripped impurities not present in the SOL. The measurements made on injected carbon provided strong and clear signals from the SOL for H-mode inter-ELM and L-mode. These measurements serve as a proxy for impurity migration. (Where do impurities eroded from the central column migrate?). However, the measured carbon flow profiles do not necessarily correspond to the actual background deuterium flow as one cannot expect full equilibration for the injected impurities. ERO [14] simulations were utilized to investigate the actual background flow.

\section{Experimental setup and data analysis}

Methane was injected from valve T3 in ASDEX Upgrade (AUG), which is located toroidally in the 
center of sector 1 and poloidally on the HFS about $13 \mathrm{~cm}$ above the midplane. The ensuing carbon emission was followed with a fast video camera equipped with appropriate filters looking vertically at the injection [15], and a spectrometer with 8 lines-of-sight running almost parallel to the magnetic field close to the injection location [6]. The 8 lines-of-sight cross the poloidal plane of the injection ranging from $\mathrm{R}=1.0856 \mathrm{~m}$ to $\mathrm{R}=1.1681 \mathrm{~m}$ giving a radial resolution of approximately 1 cm. The charge states CII $(\mathrm{C}+1,514 \mathrm{~nm})$ and CIII $(\mathrm{C}+2,465 \mathrm{~nm})$ were followed. The experimental setup is shown in figure $1(a-b)$.

The spectrometer gives the line integrated emission as well as the Doppler shift (flow velocity) and broadening of the impurity emission lines. The Doppler shift and broadening were calculated from the observed spectra using the following procedure. For every time frame and every spectroscopic line-of-sight, the magnetic field where the line-of-sight crosses the poloidal plane of the injection is evaluated using the magnetic equilibrium reconstruction. The magnetic field and geometry data is then given to the xpaschen code [16] that gives the Zeeman components of the desired CII and CIII transitions, their expected central wavelength and relative strength. This spectrum is then Doppler shifted and broadened to best match the experimentally observed spectrum. An example is shown in figure $1(d)$.

To understand how the measured CII and CIII data couples to the background deuterium flow, ERO simulations were utilized. The background flow profile was varied to give the observed carbon flow. The ERO code [14] follows neutrals and full-orbit charged particles in a given magnetic field and plasma background. ERO includes a methane break up model and atomic reactions for carbon. Friction with the background flow is accounted for by a Fokker-Planck Coulomb collision operator. The methane is injected at the position of the valve and the test particles are followed until they exit the simulation area shown in figure 1. The initial distribution is assumed Maxwellian with an initial energy of $0.05 \mathrm{eV}$ corresponding to $113{ }^{\circ} \mathrm{C}$. The simulations in this paper used a radial diffusion 
coefficient of $1 \mathrm{~m}^{2} / \mathrm{s}$. The plasma-wall-interaction models in ERO are not used in this work. This is motivated by the fact the magnetic field lines run nearly parallel to the wall at the inner midplane. Temperature gradient forces are neglected in these ERO simulations. Electric fields are neglected in this work. It should be noted that the ExB-drift can be large but it should be nearly perpendicular to the spectroscopic lines-of-sight for this experimental setup. It is thus assumed that the friction with the background flow is the dominant factor for carbon transport in the main SOL, which is a common assumption (see e.g. [2][10][17]). However, if this is not the case, the deuterium flow profile obtained by ERO simulations is likely not correct.

Addressing the question what background conditions are required for ERO to reproduce the observed experimental data, the plasma profiles in the HFS SOL were varied iteratively. For this purpose, new synthetic spectroscopy and camera diagnostics with the ability to match the experimental geometry were implemented into the code. This work will be reported in future publication. The plasma profiles were assumed not to vary poloidally while the radial profiles of the density, temperature and flow velocity were then varied to best match the experimental data. The profiles for density and temperature were assumed to be described by exponential fall-off characterized by a value at the separatrix and a fall-of length. The value at the separatrix and the fall-off length were varied in the narrow range suggested by plasma diagnostics at the low field side. The radial flow profile was controlled by varying the Mach number across the SOL. Several profiles were tested including profiles inspired by C-Mod HFS deuterium flow measurements [13]. The fitting procedure was done by hand at this point. Work is under way to automatize the procedure.

This paper focuses on data gathered in AUG discharges 26569 - 26571, 27735, and 27737. The discharges 26569-26571 were similar high density H-mode (electron density $=9.44 \times 10^{19} 1 / \mathrm{m}^{3}$, total heating power $=8.159 \mathrm{MW})$. The spectrometer central wavelength and the fast camera filter were 
changed during consecutive discharges to gather CII and CIII data from similar plasma conditions. Discharges 26569 to 26571 had a large clearance between the separatrix and the inner wall. With 7 to 8 spectroscopic lines-of-sight inside the SOL, these discharges are ideal for modeling the methane injection and extensive ERO simulations have been carried out to try to disentangle the actual background deuterium flow from the observed CII and CIII data. Discharge 27735 was an Lmode density ramp and 27737 was a H-mode density ramp. The line-integrated density was varied from $2 \times 10^{19} 1 / \mathrm{m}^{2}$ to $8 \times 10^{19} 1 / \mathrm{m}^{2}$ for the L-mode discharge and $2 \times 10^{19} 1 / \mathrm{m}^{2}$ to $10 \times 10^{19} 1 / \mathrm{m}^{2}$ for the H-mode case in the period with active methane injection. Spectroscopic data was gathered for CIII.

\section{Results}

Figure 2 shows the observed inter-ELM (between ELMs) HFS SOL CII and CIII flow profiles observed in discharges 26569-26571. In region A of figure 2, both the CII and CIII flows are directed towards the inner divertor (positive values) with velocities up to $7 \mathrm{~km} / \mathrm{s}$ for CIII in the far SOL. Both the CII and CIII flows are close to zero in the transition from region A to B, and the CIII flow is negative while the CII flow is close to zero, given experimental uncertainties, in region B. This suggests a background deuterium flow with a positive flow velocity in region A and negative in region $\mathrm{B}$. With high temperatures expected in region $\mathrm{B}, \mathrm{CII}$ is too short lived to equilibrate with the deuterium flow while CIII is not.

Extensive ERO simulations were carried out for discharges 26569-26571 to investigate this picture. The flow profile was varied to match the observed CII and CIII data, as were also the density and temperature profiles in the range suggested by LFS Thomson scattering diagnostics. To a quite high accuracy, the emission profiles seen in figure 4 were only affected by the assumed density and temperature profiles. Therefore, density and temperature were determined prior to determining the flow profile. The plasma background shown in figure 3 provided the best match to experimental data from several tested backgrounds. Comparison between the actual and synthetic ERO 
diagnostics for this case is shown in figure 4 for spectroscopy and figure 5 for the camera. The emission intensity is normalized both for the spectrometer and fast camera as the exact amount of injected methane is not known.

It was observed that the qualitative shape of the flow velocity profile needed to reproduce the measured CII and CIII data is not sensitive to the assumed temperature and density profiles. However, the absolute magnitude of the background flow is. Assuming the temperature profile shown in figure 3, the ERO simulations suggest background deuterium flow profiles with velocities up to $15 \mathrm{~km} / \mathrm{s}$ (Mach-0.5) in the far SOL. The used deuterium flow profile peaks around $5 \mathrm{~cm}$ from the separatrix while no such peak is observed in the CII and CIII flow profiles. There are several reasons for this. The temperature in the far SOL is lower compared to the peak position meaning better equilibration for CII and CIII there. Anomalous radial diffusion used in the ERO simulations tend to smooth out the CII and CIII flow profiles. As the carbon ions diffuse radially, they experience the deuterium flow from various radial locations. Also, the spectrometer observes the line-averaged flow velocity of carbon. These $3 \mathrm{D}$ effects become noticeable if the carbon plume is spatially extended. These effects make it difficult to disentangle the deuterium flow velocity and the profile shown in figure 3 should be considered a plausible assumption. However, the CIII flow is typically in the direction of the deuterium flow.

Besides the density and temperature profiles at the measurement location, the obtained deuterium flow profile depends on several other factors. The sensitivity on various parameters were tested by running ERO simulations on the best matching case. The density and temperature in the far SOL is not clear from measurements. ERO simulations were carried out varying the far SOL density and temperature but the effects were small - assuming the far SOL density and temperature are low compared to the near SOL values. This is because most processes of interest (e.g. acceleration of carbon ions) happen closer to the separatrix. The diffusion coefficient, assumed $1.0 \mathrm{~m}^{2} / \mathrm{s}$, was also 
varied in the typical range of $0.5-2.0 \mathrm{~m}^{2} / \mathrm{s}$ but the effects on the results were small. Higher values for the diffusion coefficient tend to widen the emission peaks observed in figure 4 . The injection energy, $0.05 \mathrm{eV}$, was varied from $0.04 \mathrm{eV}\left(36^{\circ} \mathrm{C}\right)$ to $0.08 \mathrm{eV}\left(346{ }^{\circ} \mathrm{C}\right)$ but the effects were small because the velocity is proportional to the square root of the energy.

It should be noted, however, that the combined effect of all uncertainties can be large. Besides the factors mentioned here, turbulence is not included in the ERO code, there are uncertainties in the atomic data, and the accuracy of the Coulomb collision operator is difficult to ascertain. (For recent work on Coulomb collisions, see e.g. [18]). It is, however, beyond the scope of this paper to analyze these in detail.

Figure 6 (a) shows the observed CIII flow velocity from the L-mode density ramp 27735 and figure 6 (b) from the H-mode density ramp 27737. The times $2.3 \mathrm{~s}, 3.1 \mathrm{~s}$, and $3.9 \mathrm{~s}$ were chosen to minimize the influence of the injection on the plasma. The time 2.3 corresponds to line-averaged density of $3.3 \times 10^{19} 1 / \mathrm{m}^{3}$ for L-mode and $3.6 \times 10^{19} 1 / \mathrm{m}^{3}$ for $\mathrm{H}$-mode. This is referred to as the low density case. The medium density case is at $3.1 \mathrm{~s}$ with $5.2 \times 10^{19} 1 / \mathrm{m}^{3}$ for L-mode and $7.8 \times 10^{19} 1 / \mathrm{m}^{3}$ for H-mode. The high density case at $3.9 \mathrm{~s}$ has $7.6 \times 10^{19} 1 / \mathrm{m}^{3}$ and $9.3 \times 10^{19} 1 / \mathrm{m}^{3}$, respectively. In contrast to the high density H-mode discharges $26569-26571\left(9.4 \times 10^{19} 1 / \mathrm{m}^{3}\right)$, the flow of CIII is towards the top of the machine in large parts of the SOL in the low density cases both in L-mode and H-mode. The high density cases, on the other hand, show a qualitatively similar CIII flow profile as measured in discharges 26569-26571.

The equilibration efficiency cannot change the direction of the carbon flow. The observed behavior could be explained by a qualitatively different deuterium flow profile in the high and low density cases. Another explanation is invoking the temperature gradient force. The friction force scales linearly with density forgetting the weak dependence in the Coulomb logarithm while the 
temperature gradient force does not depend on density. The mean ionization time is inversely proportional to the density. Considering a simple model where the observed CIII flow velocity is proportional to the force multiplied by the time it takes to ionize to higher charge states, the net effect to the observed flow velocity is that higher densities do not actually increase the coupling with the deuterium flow - but rather reduces coupling to the temperature gradient force. It is possible we are seeing the effect of the temperature gradient force pushing the injected carbon away from the inner divertor at low densities while the friction force dominates at high densities. However, besides affecting coupling to the friction force and the temperature gradient force, the changing plasma density affects SOL conditions and can change the flow profile in the SOL and also the temperature gradients.

At this stage, no conclusions can be drawn from the density scans regarding the background deuterium flow. However, the direction and magnitude of the injected carbon serves as a proxy for impurity migration. These measurements indicate that impurities eroded from the central column tend to travel more towards the top of the machine in low density L-mode and H-mode plasmas and towards the inner divertor in high density plasmas.

\section{Discussion and conclusions}

The flow of injected CII and CIII was measured for varying densities in L-mode and H-mode. In the high density conditions, the flow of carbon was primarily towards the lower inner divertor with velocities up to $10 \mathrm{~km} / \mathrm{s}$ in the far SOL for CIII and a shear across the SOL. The results for low density cases show carbon flowing to the top of the machine. Temperature gradient forces are suspected. However, this can be confirmed only by combining these measurements to actual deuterium flow measurements or temperature gradient measurements. No qualitative difference for carbon flow was observed with this method for L-mode and inter-ELM H-mode. ELMs can affect the flow of carbon. 
The ERO simulations for the high-density case suggest that the observed CII and CIII flow velocities can be explained using deuterium flow velocities up to $15 \mathrm{~km} / \mathrm{s}(\sim \mathrm{Mach}-0.5)$ in the far SOL and a reversal close to the separatrix. The best matching deuterium flow profile is dependent on the assumed radial temperature profile, but qualitatively always similar with a high flow on the far SOL. Finally, the effects of the temperature gradient force cannot be completely ruled out for the high-density case.

\section{Acknowledgements}

This work, supported by the European Communities under the contract of Association between EURATOM/Tekes, was carried out within the framework of the Task Force on Plasma Wall Interactions of the European Fusion Development Agreement. The views and opinions expressed herein do not necessarily reflect those of the European Commission. This work was funded by Academy of Finland project numbers SA-253222 and SA-134924.

\section{References}

[1] J.D. Strachan et al. Nucl. Fusion 44 (2004) 772

[2] A.G. McLean et al. J. Nucl. Mater. 337-339 (2005) 124

[3] N. Tsois et al. J. Nucl. Mater. 266 (1999) 1230-1233

[4] J. Gafert et al. Plasma Phys. Control. Fusion 39 (1997) 1981

[5] V. Rohde et al. J. Nucl. Mater. 241 (1997) 712-715

[6] T. Puetterich el al. Nucl. Fusion (2012) submitted

[7] E. Viezzer et al. Rev.. Sci. Instrum. (2012) submitted

[8] K. Krieger et al. J. Nucl. Mater. 415 (2011) S297-S300

[9] P. Petersson et al. P1-092 PSI2012 Conference, Aachen, Germany (2012)

[10] J. Miettunen et al. Nucl. Fusion 52 (2012) 032001 
[11] S. Gangadhara et al. J. Nucl. Mater. 290-293 (2001) 598-603

[12] S. Gangadhara et al. J. Nucl. Mater. 313-316 (2003) 1167-1172

[13] B. LaBombard et al. Nucl. Fusion 44 (2004) 1047

[14] A. Kirschner et al. Nucl. Fusion 40 (2000) 989

[15] T. Lunt et al. Nuclear Instruments and Methods in Physics Research Section A 623 (2010) 812

[16] A. Pospieszczyk Physica Scripta 2005 (2005) 71

[17] W.R. Wampler et al J. Nucl. Mater. 363 (2007) 72

[18] R. Alexandre et al. Annales de l'Institut Henri Poincare (C) Non Linear Analysis 21 (2004) 61 
Figure 1. Toroidal (a) and poloidal (b) projection of the experimental setup and examples of experimental data from the video camera (c) and spectrometer (d). The AUG wall structure is indicated by thick black lines, the ERO simulation box is marked by thick black lines. The thin blue lines running trough the simulation are the 8 spectroscopic lines-of-sight. The green translucent pyramid is the approximate view cone of the camera. The inter-ELM time averaged emission cloud for CII (514 nm) and the inner and outer strikes lines can be seen in in sub-figure (c).

Figure 2. CII and CIII radial flow velocity profiles from the Doppler shift from \#26569 and \#26570. Positive velocities are towards the inner lower divertor. The x-axis is marked with three regions, A, $\mathrm{B}$, and $\mathrm{C}$ (core plasma) discussed in section 3 . The error bars represent $95 \%$ confidence limits.

Figure 3. The plasma background used in the best matching ERO simulations for (a) density, (b) temperature, and (c) flow velocity. The dashed line is the Mach number of the flow. Blue scatter is experimental data from the LFS Thomson scattering diagnostics mapped to the HFS.

Figure 4. Comparison between spectroscopic and synthetic ERO diagnostics for the best matching inter-ELM case. The emission maxima are normalized to one. The CII flow velocity for the core missing due to lacking statistics there. The error bars represent $95 \%$ confidence limits.

Figure 5. Comparison of synthetic ERO camera to actual camera for the best matching inter-ELM case. The emission maximum is normalized to one. The contour is for ERO data for the value 0.3 . This is also plotted on the experimental image.

Figure 6. Measured CIII flow from (a) L-mode discharge 27735 and (b) H-mode discharge 27737 for low density (2.3 s), medium density ( $3.1 \mathrm{~s})$, and high density cases ( $3.9 \mathrm{~s})$ described in section 3 . 
Figure 1.

$75 \mathrm{~mm}$ wide

$75 \mathrm{~mm}$ high

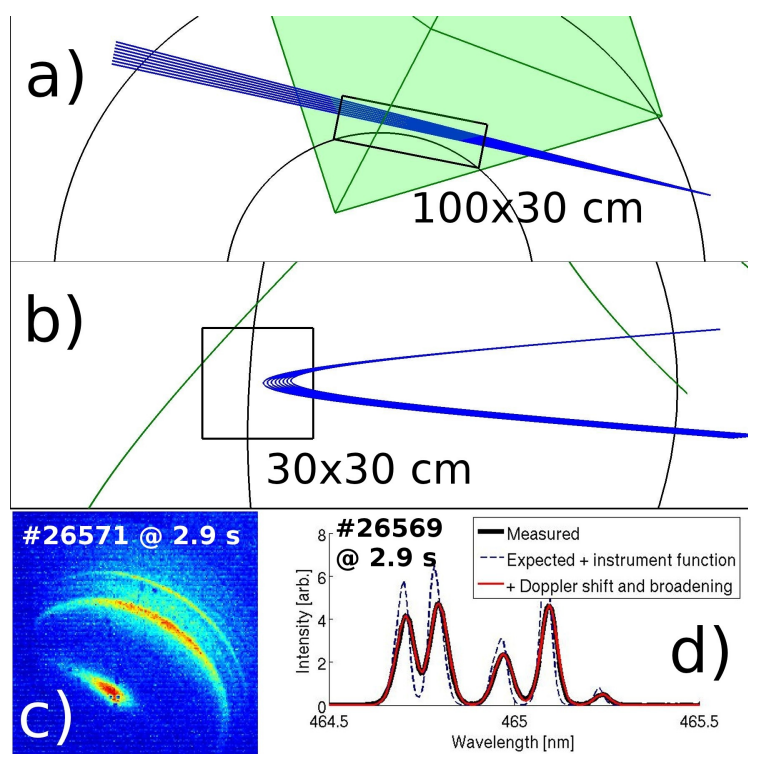


Figure 2.

$75 \mathrm{~mm}$ wide

$50 \mathrm{~mm}$ high

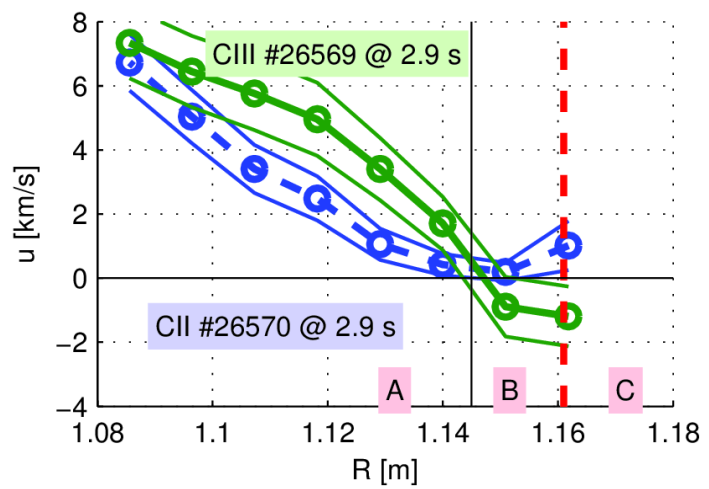


Figure 3.

$75 \mathrm{~mm}$ wide

$75 \mathrm{~mm}$ high

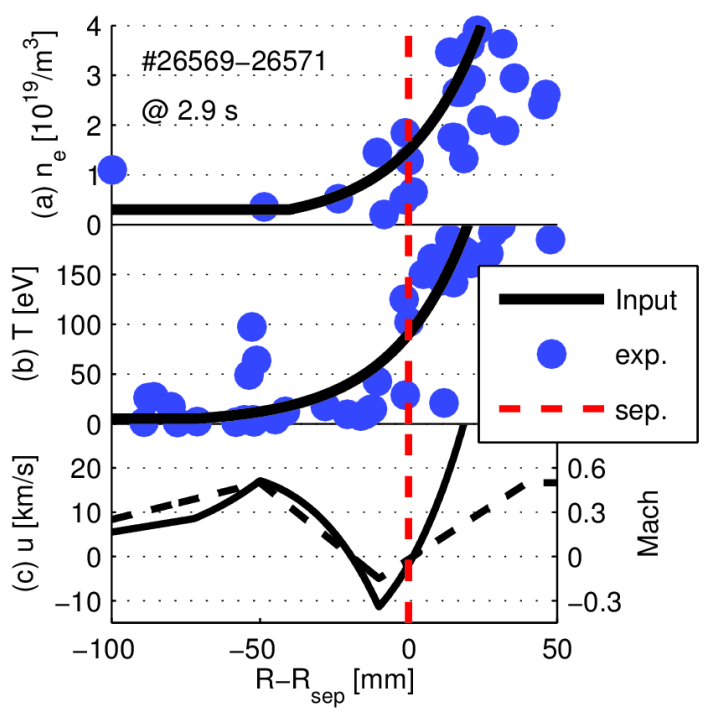


Figure 4.

$75 \mathrm{~mm}$ wide

$50 \mathrm{~mm}$ high

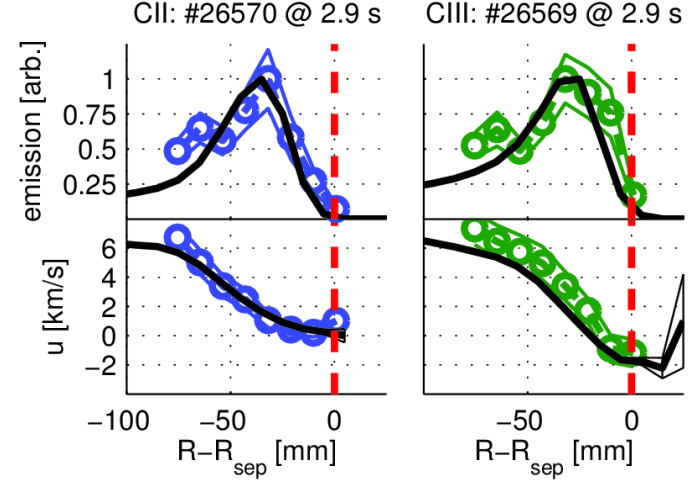


Figure 5

$75 \mathrm{~mm}$ wide

$50 \mathrm{~mm}$ high

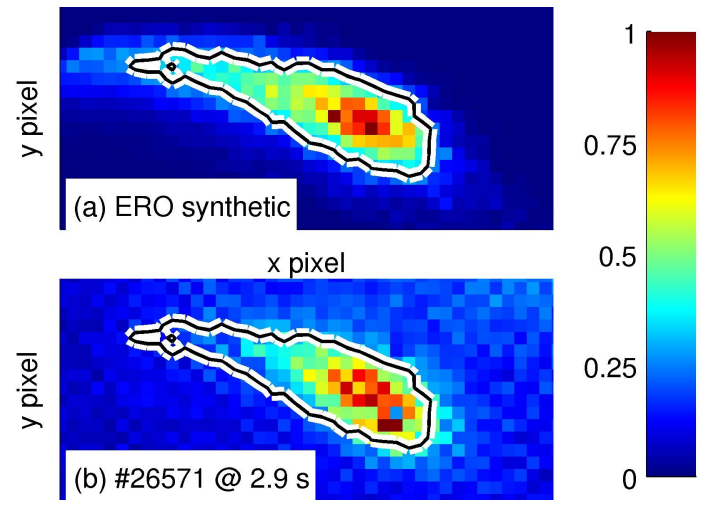


Figure 6

$75 \mathrm{~mm}$ wide

$50 \mathrm{~mm}$ high

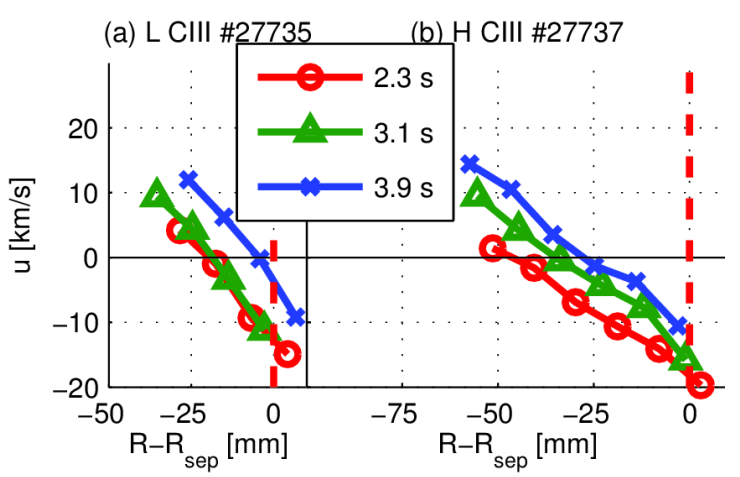

